\title{
The Dynamic Multi-Task Supply Chain Principal-Agent Analysis
}

\author{
Shanliang LI ${ }^{1,2}$, Chunhua WANG ${ }^{3}$, Daoli ZHU ${ }^{1}$ \\ ${ }^{1}$ Management School, Fudan University, Shanghai, China; ${ }^{2}$ Management School, Soochow University, Suzhou, China; ${ }^{3}$ Information \\ School, Shanghai Ocean University, Shanghai, China. \\ Email: Lisl@fudan.edu.cn
}

Received August 18, 2009; revised September 25, 2009; accepted November 5, 2009.

\begin{abstract}
In the supply chain by the composition of the supplier and the retailer, the supplier offers products to the retailer for sales while the retailer affects the sales outcome by his effort which is divided into two dimensions. One is for the short-term sales task and the other is for the long-term sales task. For the long-term development of the enterprise, the supplier wants to inspire the retailer to make more effort for the long-term task. However, due to the asymmetric information, the supplier can't observe the retailer's action and the moral hazard will come into being. To deal with this problem, we construct the dynamic multi-task supply chain principal-agent model, by which we analyze the impact of the information asymmetry to the supply chain contract. Furthermore, by comparing the contracts between the single-term multi-task and two-term multi-task, we have analyzed their different effect on the commission rate.
\end{abstract}

Keywords: Supply Chain Management, Multi-task Principal-agent, Dynamic Incentive, Moral Hazard

\section{Introduction}

In the supply chain system, there exists the conflict between the local interests of the supply chain members and the overall performance of the supply chain, which leads to the system inefficiency. At present, one of the most important research areas of supply chain is to design the suitable coordination mechanism in order to obtain the global optimization of the supply chain performance. In this process, the information plays a very important position. As the supply chain members tend to hide their private information to maintain information superiority, this will lead to "Adverse Select" and "Moral Hazard” in various fields [1].

In the recent decade, scholars have studied on the issue of the supply chain coordination from various angles. These studies can be roughly divided into two categories. One is to resolve the "double marginalization" problem by contract design in the situation of the full information [2-4]. Such contracts do not involve the information incentive. The other is to study the supply chain incentive problem in the situation of the asymmetric information. Corbett etc. studied that the optimal quantity discounts incentive contract between the supplier and the retailer [5]. Basu etc. studied the incentive issues of the sales force under asymmetric information based on agency theory [6]. Lal etc. [7,8] and Chen [9] extended the above studies. Many Chinese academics are also carried out research on this issue [10-14]. For the supply chain coordination, the author's research team had a systemic research on the issue earlier. Some relevant research results have been published [15-22]. This paper is the important one of the systemic study. In the simple principal-agent model, the agent is engaged in one job and the agent's effort is one-dimensional. However in many cases of the real life, agents are engaged in the job of more than one. Or, even if there is only one job, it involves more than one dimension. Furthermore, it exits conflict in the distribution of the same agent's energy between the different jobs. To deal with this problem, we construct the dynamic multi-task supply chain principal-agent model, by which we analyze the impact of the information asymmetry to the supply chain contract. Furthermore, by comparing the contracts between the single-term multi-task and two-term multi-task, we analyzed their different effect on the commission rate.

\section{Assumptions and Parameters Set}

We make the following assumptions for the tractable analysis. Considering a Stackelberg model between a supplier $S$ who is the principal and a retailer $R$ who is the agent, the supplier offers the retailer products to sale and pays the retailer according to sales outcome which is 
affected by the retailer's effort and the random factors. Set $B_{1}\left(a_{1}, a_{2}\right)$ is the retailer's expected profit whose ownership belongs to the supplier. $a_{1}$ denotes the retailer's effort for the short sales goal. $a_{2}$ denotes the retailer's effort for the long sales goal. $C\left(a_{1}, a_{2}\right)$ denotes the cost of the retailer's effort, satisfying $\frac{\partial C}{\partial a_{1,2}} \geq 0, \frac{\partial^{2} C}{\partial a_{1,2}^{2}} \geq 0$, i.e. the cost of the effort increases and the marginal cost increases. For the simplicity, Assume $C\left(a_{1}, a_{2}\right)=\frac{a_{1}^{2}}{2}+\frac{a_{2}^{2}}{2}$. The supplier can't observe $a_{1}$ and $a_{2}$, but can observe and verify the sales outcome $x$, which is affected by the retailer's effort together with the random variables, denoted by $x=\mu\left(a_{1}, a_{2}\right)+\theta$, where $\mu\left(a_{1}, a_{2}\right)$ is the output function of the effort, satisfying $\frac{\partial \mu}{\partial a_{1,2}} \geq 0$, which means the marginal sales outcome of the effort is positive. i.e. more efforts mean more sales; $\frac{\partial^{2} \mu}{\partial a_{1,2}^{2}} \leq 0$, which means marginal sales outcome decrease (When the equal sign is set up, marginal sales unchanged). Set $\theta^{T}=\left(\theta_{1}, \theta_{2}\right)$, which is the random variable of Normal distribution and satisfy $N \sim\left(0, \sigma_{1}^{2} ; 0, \sigma_{2}^{2} ; r\right)$; Set $x^{T}=\left(x_{1}, x_{2}\right)$, For the sake of simplifying the calculating, assume that $x_{1}=\mu\left(a_{1}\right)+\theta_{1}=a_{1}+\theta_{1} ; \quad x_{2}=\mu\left(a_{2}\right)+\theta_{2}=a_{2}+\theta_{2}$. i.e. different efforts result in different information (However, different information may be relevant if $\theta_{1}$ and $\theta_{2}$ are relevant. ): $x_{1}$ reflects $a_{1}, x_{2}$ reflects $a_{2}$. The ownership of the sales profits belongs to the supplier, and the supplier offers the linear salary to pay the retailer.

$$
s(x)=\alpha+\beta^{T} x=\alpha+\beta_{1} x_{1}+\beta_{2} x_{2}
$$

where $s(x)$ is the wage paid to the retailer if the total sales outcome is $x . \alpha$ denotes the one-off wealth transfer which doesn't affect the incentive intension (Called Salary); $\beta^{T}=\left(\beta_{1}, \beta_{2}\right)$ which denotes the incentive intension (Called Commission Rate), that means if $x$ increase by one unit, the wage of the retailer increased by $\beta$ unit.

\section{The Single-Stage Multi-Task Model}

In the single-stage model, the supplier offers a one-time wage schedule, $s(x)$, according to which the retailer is rewarded. Assume the supplier is risk-neural, the expected utility function is as follows:

$$
\begin{gathered}
E U_{S}=B\left(a_{1}, a_{2}\right)-\alpha-E \beta^{T} \mu\left(a_{1}, a_{2}\right) \\
=B\left(a_{1}, a_{2}\right)-\alpha-\beta_{1} a_{1}-\beta_{2} a_{2}
\end{gathered}
$$

Assume the retailer is risk-averse, and the utility is that $V(x)=-e^{-\rho x}$, where $\rho$ is the risk aversion coefficient. When $\rho=0$, the retailer is risk-neural. When $\rho>0$, the retailer is risk-averse. When $\rho<0$, the retailer is risk preference. The retailer's expected utility is as follows:

$$
E U_{R}=E V\left(s(x)-C\left(a_{1}, a_{2}\right)\right)
$$

To make the analysis simple, we use the certainty equivalent (CE) instead of the expected utility of the retailer [18].

$$
C E_{R}=\alpha+\beta^{T} \mu\left(a_{1}, a_{2}\right)-\frac{1}{2} \rho \beta^{T} \Sigma \beta-C\left(a_{1}, a_{2}\right)
$$

where $\alpha+\beta^{T} \mu\left(a_{1}, a_{2}\right)$ is the expected wage, $\rho$ is risk aversion coefficient, $\beta^{T} \Sigma \beta$ is the income variance, $\rho \beta^{T} \Sigma \beta / 2$ is the risk cost. $\Sigma$ is the covariance matrix of $\theta_{1}$ and $\theta_{2}$, denoted by $\Sigma=\left(\begin{array}{l}\delta_{1}^{2}, r \delta_{1} \delta_{2} \\ r \delta_{1} \delta_{2}, \delta_{2}^{2}\end{array}\right)$.

The supplier is the leader in the Stackelberg model, who has first-step advantage in the game. However, when he/she pursuits the profit maximization, he/she must consider the incentive compatibility constraint and participation constraint. Thus, the principal-agent model between the supplier and the retailer can be rewritten as the following optimization programming.

$$
\text { (P) } \underset{\alpha, \beta, a}{\operatorname{Max}} E U_{S}=B\left(a_{1}, a_{2}\right)-\alpha-\beta_{1} a_{1}-\beta_{2} a_{2}
$$

s.t. (IR) $C E_{R}=\alpha+\beta_{1} a_{1}+\beta_{2} a_{2}$

$$
-\frac{1}{2} \rho\left(\beta_{1}, \beta_{2}\right)\left(\begin{array}{l}
\delta_{1}^{2}, r \delta_{1} \delta_{2} \\
r \delta_{1} \delta_{2}, \delta_{2}^{2}
\end{array}\right)\left(\begin{array}{l}
\beta_{1} \\
\beta_{2}
\end{array}\right)-\frac{a_{1}^{2}}{2}-\frac{a_{2}^{2}}{2} \geq 0
$$

$$
\text { (IC) } \arg \max _{a_{1}, a_{2}} C E_{R}
$$

where (6) is participation constraint (IR), and (7) is incentive compatibility constraint (IC).

\subsection{The Full Information Benchmark}

In this section, let's begin with the full information case where the retailer's effort is observable and verifiable. Then the supplier can assign an effort level to the retailer by designing a forcing contact. Under this condition, the incentive compatibility (7) is invalid and we only consider the participation constraint (6), which is binding. Namely, $(P)$ can be rewritten as follows:

$$
\begin{array}{ll}
\left(P^{\prime}\right) & \operatorname{Max}_{\alpha, \beta, a} U_{S}=B\left(a_{1}, a_{2}\right)-\alpha-\beta_{1} a_{1}-\beta_{2} a_{2} \\
\text { s.t. } & C E_{R}=0
\end{array}
$$


Solving $\left(P^{\prime}\right)$, we can obtain that:

$$
\begin{aligned}
& \frac{\partial B\left(a_{1}, a_{2}\right)}{\partial a_{1}}=\frac{\partial C\left(a_{1}, a_{2}\right)}{\partial a_{1}}=a_{1} \\
& \frac{\partial B\left(a_{1}, a_{2}\right)}{\partial a_{2}}=\frac{\partial C\left(a_{1}, a_{2}\right)}{\partial a_{2}}=a_{2}
\end{aligned}
$$

The Equation (9) is the class condition of the Pareto optimality: the expected marginal profit of the effort is equal to the expected marginal cost. That is similar to the single-task case. We have the following conclusion.

Proposition 1: Under the condition of full information, by designing the linear incentive contract, the game between the supplier and the retailer can achieve the Pareto optimality when the retailer has multi-dimensional effort.

\subsection{The Asymmetric Information Case}

Generally, the supplier can't observe the retailer's action $a$, and only can observe outcome $x$. In this case, the incentive compatibility constraint (7) is valid. Substituting (7) by the first-order condition, we can obtain the equivalent programming. i.e. (7) is equal to that

$$
\begin{aligned}
& a_{1}=\beta_{1} \\
& a_{2}=\beta_{2}
\end{aligned}
$$

Solving the model $(P)$

$$
\underset{\alpha, \beta}{\operatorname{Max}} B\left(a_{1}, a_{2}\right)-\left[\frac{1}{2} \rho\left(\beta_{1}, \beta_{2}\right)\left(\begin{array}{l}
\delta_{1}^{2}, r \delta_{1} \delta_{2} \\
r \delta_{1} \delta_{2}, \delta_{2}^{2}
\end{array}\right)\left(\begin{array}{l}
\beta_{1} \\
\beta_{2}
\end{array}\right)+\frac{a_{1}^{2}}{2}+\frac{a_{2}^{2}}{2}\right]
$$

Substituting by (10), we get

$$
\underset{\alpha, \beta}{\operatorname{Max}} B\left(\beta_{1}, \beta_{2}\right)-\left(\frac{1}{2} \rho\left(\beta_{1}^{2} \delta_{1}^{2}+2 r \beta_{1} \beta_{2} \delta_{1} \delta_{2}+\beta_{2}^{2} \delta_{2}^{2}\right)+\frac{\beta_{1}^{2}}{2}+\frac{\beta_{2}^{2}}{2}\right)
$$

Solve the first order derivative of (13), (14) about $\beta_{1}$, obtain

$$
\frac{\partial B}{\partial \beta_{1}}-\left(\frac{1}{2} \rho\left(2 \beta_{1} \delta_{1}^{2}+2 r \beta_{2} \delta_{1} \delta_{2}\right)+\beta_{1}\right)=0
$$

Solving the above equation, we obtain that

$$
\beta_{1}=\frac{\partial B / \partial \beta_{1}-\rho r \beta_{2} \delta_{1} \delta_{2}}{1+\rho \delta_{1}^{2}}
$$

Similarly, we get

$$
\beta_{2}=\frac{\partial B / \partial \beta_{2}-\rho r \beta_{1} \delta_{1} \delta_{2}}{1+\rho \delta_{2}^{2}}
$$

By (13), (14), we get the following conclusion.

Proposition 2: When $\rho=0$, the retailer is risk-neural, then $\beta_{i}=\frac{\partial B}{\partial \beta_{i}}=\frac{\partial C}{\partial a_{i}}=a_{i}(i=1,2)$, which means the game can get the Pareto optimization just as the full information case. When $\rho>0, \beta_{i} \quad(i=1,2)$ is in inverse ratio with $\rho$, the risk aversion coefficient will reduce the incentive intensity $\beta_{i} ; \beta_{i}(i=1,2)$ is in inverse ratio with variance $\delta_{i}^{2} \quad(i=1,2)$; in inverse ratio with the Covariance $r \delta_{1} \delta_{2}$, i.e. the random factors also reduce the incentive intensity of $\beta_{i} . \beta_{1}$ is also in inverse ratio with $\beta_{2}$. More $\beta_{2}$ means less $\beta_{1}$, and vice versa.

\section{Two-Stage Multi-Task Game Model}

In the two-stage multi-task model, suppose the retailer's effort $a_{2}$ for the long task in the first stage will affect the profit in the second stage of the supply chain. Set $B_{1}\left(a_{1}, a_{2}\right)$ denotes the expected effort profit of the first stage of the retailer, $B_{2}\left(a_{21}, a_{22}, a_{2}\right)$ denotes the expected profit of the second stage. Where $a_{21}$ and $a_{22}$ denote the effort for the short task and long task respectively. Because the effort $a_{2}$ in the first stage will affect the profit in the second stage, it will be the variable of the output function of the second stage. The ownership of $B_{1}\left(a_{1}, a_{2}\right)$ and $B_{2}\left(a_{21}, a_{22}, a_{2}\right)$ belongs to the supplier, the supplier offers the wage schedule according to the two-stage outcome. Similarly to the assumption of onestage, the observed outcome in the second stage is that

$$
x 2^{T}=\left(x_{1}, x_{2}, x_{3}, x_{4}, x_{5}\right)
$$

where $x_{1}=a_{1}+\theta_{1}, \quad x_{2}=a_{2}+\theta_{2}, \quad x_{3}=a_{21}+\theta_{3}$, $x_{4}=a_{22}+\theta_{4}, \quad x_{5}=a_{2}+\theta_{5}$. Assume $\theta^{\prime T}=\left(\theta_{3}, \theta_{4}, \theta_{5}\right)$, which is the random variable in the second stage, Independent with $\theta^{T}=\left(\theta_{1}, \theta_{2}\right)$, the random variable in the first stage. The supplier offers the two-stage payoff contract according the observed outcome as follows.

$$
\begin{aligned}
& s_{1}(x)=a_{1}+\beta_{1} x_{1}+\beta_{2} x_{2} \\
& s_{2}(x)=\alpha_{2}+\beta_{3} x_{3}+\beta_{4} x_{4}+\beta_{5} x_{5}
\end{aligned}
$$

The supplier's expected utility is that:

$$
E U_{S}=B_{1}\left(a_{1}, a_{2}\right)-E s_{1}(x)+B_{2}\left(a_{21}, a_{22}, a_{2}\right)-E s_{2}(x)
$$

Now, the certainty equivalent of the retailer in the first stage and the second stage is that

$$
\begin{array}{r}
C E_{R}{ }^{1}=\alpha_{1}+\beta_{1} a_{1}+\beta_{2} a_{2}-\frac{1}{2} \rho \beta^{T} \Sigma \beta-\frac{a_{1}^{2}}{2}-\frac{a_{2}^{2}}{2} \\
C E_{R}{ }^{2}=\alpha_{2}+\beta_{3} a_{21}+\beta_{4} a_{22}+\beta_{5} a_{2}-\frac{1}{2} \rho \beta_{21}^{T} \Sigma_{1} \beta_{21}-\frac{a_{21}^{2}}{2}-\frac{a_{22}^{2}}{2}
\end{array}
$$


where $\Sigma_{1}$ is covariance matrix $\theta_{3}, \theta_{4}$ and $\theta_{5}$. Denoted by

$$
\Sigma_{1}=\left(\begin{array}{l}
\sigma_{1}^{2}, r_{1} \sigma_{1} \sigma_{2}, r_{2} \sigma_{1} \sigma_{3} \\
r_{1} \sigma_{1} \sigma_{2}, \sigma_{2}^{2}, r_{3} \sigma_{2} \sigma_{3} \\
r_{2} \sigma_{1} \sigma_{3}, r_{3} \sigma_{2} \sigma_{3}, \sigma_{3}^{2}
\end{array}\right)
$$

For obtaining the retailer's optimal effort of the second stage, solve $\underset{a_{21}, a_{22}}{\arg \max } C E_{R}{ }^{2}$, and get

$$
\begin{aligned}
& a_{21}=\beta_{3} \\
& a_{22}=\beta_{4}
\end{aligned}
$$

Considering the participation constraint and incentive compatibility constraint, the supplier need solve the following programming:

$\left(P^{\prime \prime}\right) \underset{\alpha, \beta}{\operatorname{MaxE}} U_{P}=B_{1}\left(a_{1}, a_{2}\right)-E_{s_{1}}+B_{2}\left(a_{21}, a_{22}, a_{2}\right)-E_{s_{2}}$

s.t. (IC) $\arg \max C E^{1}+C E^{2}$

(IR) $C E^{1}+C E^{2} \geq 0$

Instead (IC) in (22) by the first-order condition and substitute by (10), (21), Solve the programming $P^{\prime \prime}$ and get

$$
\begin{array}{r}
B_{1}\left(a_{1}, a_{2}\right)-\left[\frac{1}{2} \rho\left(\beta_{1}^{2} \delta_{1}^{2}+2 r \beta_{1} \beta_{2} \delta_{1} \delta_{2}+\beta_{2}^{2} \delta_{2}^{2}\right)+\frac{\beta_{1}^{2}}{2}+\frac{\beta_{2}^{2}}{2}\right] \\
+B_{2}\left(a_{21}, a_{22}, a_{2}\right)-{ }_{3}^{2} \sigma_{1}^{2}+r_{1} \beta_{3} \beta_{4} \sigma_{1}\left[\frac { 1 } { 2 } \left(\beta \sigma_{2}+r_{2} \beta_{3} \beta_{5} \sigma_{1} \sigma_{3}\right.\right. \\
+r_{1} \beta_{3} \beta_{4} \sigma_{1} \sigma_{2}+\beta_{4}^{2} \sigma_{2}^{2}+r_{3} \beta_{4} \beta_{5} \sigma_{2} \sigma_{3} \\
\left.\left.+r_{2} \beta_{3} \beta_{5} \sigma_{1} \sigma_{3}+r_{3} \beta_{4} \beta_{5} \sigma_{2} \sigma_{3}+\beta_{5}^{2} \sigma_{3}^{2}\right)+\frac{\beta_{3}^{2}}{2}+\frac{\beta_{4}^{2}}{2}\right]
\end{array}
$$

Solve $\beta_{1}$ by derivate (23) about $\beta_{1}$ and get

$$
\begin{gathered}
\partial B_{1} / \partial \beta_{1}{ }^{+}{ }^{\partial B_{2}} / \partial \beta_{1}-\beta_{1}\left(1+\rho \delta_{1}^{2}\right)-\rho r \beta_{2} \delta_{1} \delta_{2}=0 \\
a_{1}=\beta_{1}=\frac{\partial B_{1} / \partial \beta_{1}{ }^{+} \partial B_{2} / \partial \beta_{1}-\rho r \beta_{2} \delta_{1} \delta_{2}}{1+\rho \delta_{1}^{2}}
\end{gathered}
$$

Solve $\beta_{2}$ by derivate (23) about $\beta_{2}$ and get

$$
\begin{gathered}
\partial B_{1} / \partial \beta_{2}+{ }^{\partial B_{2}} / \partial \beta_{2}-\beta_{2}\left(1+\rho \delta_{2}^{2}\right)-\rho r \beta_{1} \delta_{1} \delta_{2}=0 \\
a_{2}=\beta_{2}=\frac{\partial B_{1} / \partial \beta_{2}+\frac{\partial B_{2} / \partial \beta_{2}-\rho r \beta_{1} \delta_{1} \delta_{2}}{1+\rho \delta_{2}^{2}}}{\text { (B) }}
\end{gathered}
$$

Because $B_{2}\left(a_{21}, a_{22}, a_{2}\right)$ doesn't involve the variable $a_{1}, \partial B_{2} / \partial \beta_{1}=0$. The Equation (13) is the same to the Equation (25). Comparing (14) with (27), because $\partial B_{2} / \partial \beta>0$, it is evident $\mathrm{B}>\mathrm{A}$. Thus, we have the following conclusion.

Proposition 3: By designing dynamic multi-task contract, the supplier can inspire the retailer to pay more effort for the long-term goal without the premise of changing the retailer's effort for the short goal. It shows that the dynamic contract design is conducive to maintain the long-term supply chain partnership.

\section{Conclusions}

The supply chain contract design is the important means of the supply chain coordination. For different environment, it will greatly improve the level of supply chain collaboration by the design of appropriate contract. In this paper, we have studied the incentive contract between the supplier and the retailer. Because of asymmetrical information, the supplier can't observe the effort level of the retailer. Therefore, the supplier can only inspire the retailer's different effort level by the incentive mechanisms design. The major study of the paper is on how to design the dynamic incentive contract to stimulate retailers to pay more efforts for the long-term under asymmetric information and multi-task environment, which has the guiding role for establishing the supply chain dynamic alliance. At the same time, our study extends the existing research results of the principal-agent. In our research work, for the sake of simplifying the technical analysis and the calculating, we focused on the second-term multi-task game. In the future, we will extend our research to multi-term multi-task model, which would be challenging and meaningful.

\section{Acknowledgements}

The authors would like to thank the referees for their helpful suggestions. The work is supported by China Postdoctoral Science Foundation under Grant No. 20080430075 and Special Grade of Financial Support from China Postdoctoral Science Foundation under Grant No. 200902199 and National Natural Science Foundation under Grant No. 70171010 .

\section{REFERENCES}

[1] W. Y. Zhang, "Game Theory and Information economics [M],” Shanghai People's Publishing House, 1997.

[2] G. Cachon, "Supply chain coordination with contracts," Handbooks in operations research and management science: Supply Chain Management, North Holland, 2003.

[3] G. Cachon and M. Lariviere, "Contracting to assure supply: 
How to share demand forecasts in a supply chain [J]," Management Science, Vol. 47, No. 5, pp. 629-646, 2001.

[4] B. Pasternack, "Optimal pricing and return policies for perishable commodities [J]," Marketing science, Vol. 4, No. 4, pp. 166-176, 1985.

[5] C. Corbett, D. Zhou, and C. Tang, "Designing supply contracts: Contract type and information asymmetry [J]," Management Science, Vol. 50, No. 4, pp. 550-559, 2004.

[6] A. Basu, R. Lal., V. Srinivasan, and R. Staelin, "Salesforce compensation plans: An agency theoretic perspective [J],” Marketing science, Vol. 4, pp. 267-291, 1985.

[7] R. Lal and V. Srinivasan, "Compensation plans for single-and multi-product salesforces: An application of the Holmstrom-Milgrom model [J]," Management Science, Vol. 39, No. 7, pp. 777-793, 1993.

[8] R. Lal and R. Staelin, "Salesforce compensation plans in environments with asymmetric information [J]," Marketing science, Vol. 5, pp. 179-198, 1986.

[9] F. Chen, "Sales-force incentives and inventory management [J]," Manufacturing \& Service Operations Management, Vol. 2, No. 2, pp. 186-202, 2000.

[10] S. Z. Bai and X. Y. Zhu, "Studyon Incentive- Mechanism of S. C MoralRisk Based on Principal-agent [J],” Logisitcis Technology, Vol. 10, 2007.

[11] X. Y. Bao and Y. Pu, "Incentive contract in reverse supply chain with asymmetric information [J]," Computer Integrated Manufacturing Systems, Vol. 9, 2008.

[12] S. P. Wu and X. Y. Xu, "Incentive mechanism for two-echelon supply chain under asymmetric information,” Computer Integrated Manufacturing Systems, Vol. 14, No. 3, 2008.

[13] C. Q. Yi and J. P. Wan, "Dynamic Principal-Agent Model with Two stage [J],” Chinese Journal of Mangement, S2, 2005.
[14] H. S. Yu, L. D. Zhao, and Y. H. Long, “An Evolutionary Game Model for Supply Chain Partnerships System Based Reputation Incentive,” Mathematics in Practice and Theory, Vol. 38, No. 4, 2008.

[15] S. L. Li and C. H. Wang, "The Analysis of the Supply Chain Incentive Contract under Asymmetric Information [C]," The 4th International Conference on WICOM: EMS2008, 2008.

[16] S. L. Li and C. H. Wang, "Linear Incentive Contract for Principal-agent Problem with Asymmetric Information and Moral Hazard [C],” 2006 IEEE Asia Pacific Conference on Circuits and Systems, 2006.

[17] S. L. Li and C. H. Wang, "The Supply Chain Incentive Contract under Double Moral-Hazard [C]," 2007 International Conference of Systems Science, Management Science and System Dynamics, 2007.

[18] S. L. Li and D. L. Zhu, "Principal-agent analysis of supply chain incentive contract with asymmetric information," Computer Integrated Manufacturing Systems, Vol. 12, 2005.

[19] S. L. Li and D. L. Zhu, "Supply Chain Linear Incentive Contract with Asymmetric Information and Moral Hazard [J],” OR Transactions, Vol. 2, 2005.

[20] S. L. Li, D. L. Zhu, and B. Wang, "Study on the Principal-agent Problem in Supply Chain,” Logistics Technology, Vol. 11, 2004.

[21] S. L. Li, M. Zuo, and D. L. Zhu, "The Analysis on the Pricipal-agent Model of a Product Line Design,” Chinese Journal of Management Science, Vol. 13, No. 1, 2005.

[22] Q. Xu, D. L. Zhu, and S. L. Li, “The Supply Chain Optimal Contract Design under Asymmetrical Information,” System Engineering-Theory \& Practice, Vol. 4, 2007. 